\title{
List of Reviewers \\ Volume 26 (2017)
}

The Editors-in-Charge wish to thank the following reviewers:

Alamaniotis Miltiadis
Alexandridis Georgios
Alizadeshani Roohallah
Amorim Renato Cordeiro de
Anagnostopoulos Christos
Antoniou Grigoris
Banjade Rajendra
Barkaoui Kamel
Boloni Ladislau
Bosco Lo Giosue
Bourbakis Nikolaos
Burris John
Camastra Francesco
Chatzilygeroudis Konstantinos
Cheng Shou-Hsiung
Ciaramella Angelo
Claro Daniela Barreiro
Deneke Wesley
Drakopoulos Georgios
Duval Beatrice
Fister Iztok, Jr.
Gao Xiao-Zhi
Gaspar Alessio
Gautam Dipesh
Georgopoulos Efstratios
Ghalwash Mohamed
Gunturi Venkata
Hao Jin-Kao
Idoumghar Lhassane

Jabbour Said
Jantzen Jan
Jegou Philippe
Kalles Dimitrios
Kanavos Andreas
Kannan S. R.
Kapellmann-Zafra Gabriel
Katakis Ioannis
Lagniez Jean-Marie
Lampropoulos Aristomenis
Lecoutre Christophe
Maharjan Nabin
Mali Amol
Maragoudakis Manolis
Maratea Antonio
Maudes Jesus
Monmarche Nicolas
Nanopoulos Alexandros
Nardone Davide
Niraula Nobal
Panangadan Anand
Papadakis George
Papadopoulos George Angelos
Papanikolaou Giannis
Patsakis Constantinos
Perikos Isidoros
Porumbel Daniel
Quyang Haibin
Rai Sanish


Riccio Angelo

Robilliard Denis

Ryu Christopher

Santipantakis George

Serraji Maria

Sgarbas Kyriakos

Staiano Antonino

Stafylopatis Andreas

Stamatatos Efstathios

Stefanescu Dan

Symeonidis Panagiotis

Theodoridis Evangelos

Tryfonopoulos Christos

Tsakalides Panagiotis

Tzanetos Alexandros
Umbrico Alessandro

Vakali Athina

Vallati Mauro

Vassiliadis Vassilios

Villar Jose Ramon

Vogel Carl

Voulgaris Zacharias

Wang Cheng-Yi

Wee G. William

Wolter Diedrich

Yu Lean

Yue Huanjing

Zelikovitz Sarah

Zhu Nanrun 